Z. klin. Chem. u. klin. Biochem.

10. Jg. 1972 , S. $112-113$

\title{
Beitrag zum Stoffwechsel von Carbamazepin
}

\author{
Von S. Goenechea und E. Hecke-Seibicke \\ Institut für Gerichtliche Mediqin (Direktor: Prof. Dr. H. Elbel) der Universitä̆ Bonn
}

(Eingegangen am 16. September 1971)

In den Urinproben zweier Patienten, die täglich 1,2 g Carbamazepin (Tegretal) nahmen, ließen sich - in Übereinstimmung mit früheren Veröffentlichungen - außer dem unveränderten Wirkstoff sieben Metaboliten dünnschichtchromatographisch nachweisen. 171 Urin der beiden Patienten wurden bei pH 3 mit Chloroform extrahiert; bei wiederholter Chromatographie des Chloroformextraktes an Kieselgel wurde ein Metabolit isoliert. Aus den spektroskopischen Befunden (UV-, IR-, Massen- und NMR-Spektren) ließ sich für diesen Metabolit die Struktur 10,11-Dihydroxy-5H-dibenzo-(b, f)-dihydroazepin-5-carbamoyl ableiten.

\section{The metabolism of carbamazapine}

After the daily administration of $1.20 \mathrm{~g}$ carbamazapine (Tegretal) to each of two patients, seven metabolites wete detected by thin layer chromatography, in addition to the unchanged starting material. This was in agreement with findings reported previously. 171 of urine from the two patients were extracted with chloroform at $\mathrm{pH} 3$. One metabolite was isolated after repeated chromatography of the chloroform extract on kieselgel. On the basis of the UV-, IR-, mass- and NMR-spectra, this metabolite was identified as 10,11-dihydroxy-5Hdibenzo-(b, f)-dihydroazepine-5-carbamoyl.

Es wird ein zentraler Angriffspunkt des Carbamazepin (5H-Dibenz [b, f] azepin-5-carboxamid) angenommen, wobei noch offen ist, inwieweit die zentralen Effekte dieses Pharmakons auf die entstandenen Metaboliten zurückzuführen sind $(1,2,3)$. Es wird ferner angenommen, daß Carbamazepin im Organismus gespeichert werden kann; es ist allerdings ungeklärt, welche Bedeutung in diesem Zusammenhang den im Harn gefundenen Stoffwechselprodukten zukommt (2).

Bisher sind im Harn und in der Duodenalflüssigkeit neben dem unveränderten Wirkstoff 7 Metaboliten dünnschichtchromatographisch nachgewiesen worden $(1,2)$. Im Liquor $(1,2,3)$ sowie im Blut (4) fand man außer dem unveränderten Pharmakon 2 Metaboliten. Bisher ist die Struktur der Stoffwechselprodukte des Carbamazepin jedoch unbekannt.

Bei unseren Untersuchungen haben wir aus dem Urin von Patienten, die in einem längeren Zeitraum Carbamazepin eingenommen hatten, einen Metaboliten isoliert und dessen chemische Struktur aufgeklärt.

\section{Arbeitsmethodik}

Extraktion des Harnes

171 Urin von 2 Patienten (Tagesdosis $1,2 \mathrm{~g}$ Carbamazepin) wurden bei $\mathrm{pH} 3 \mathrm{mit}$ Chloroform extrahiert. Die angegebene Urinmenge wurde in einem Zeitraum von 4 Wochen gewonnen. Nach Destillation des Chloroforms betrug der erhaltene Rückstand 6,1 g.

Dünnschichtchromatographie

Es wurden Platten von $20 \times 20 \mathrm{~cm}$ benutzt, die mit Kieselgel G nach STAHL beschichtet waren. Es wurde die Technik der zweidimensionalen Trennung angewandt.

Fließmittel: Chloroform/Methanol 90:10 $(\mathrm{v} / \mathrm{v})$ in beiden Richtungen.

Nachıeisreagenz: 70proz. Perchlorsäure (2,3). Die Platten wurden vor dem Besprühen an der Luft (etwa $30 \mathrm{Min}$.) getrocknet.. Nach Behandlung mit Perchlorsäure wurden sie bei $140^{\circ}$ etwa $20 \mathrm{Min}$. erhitzt. Die Auswertung erfolgte unter der UV-Lampe.

\section{Säulenchromatographie}

Zur Auftrennung des Chloroformextraktes wurde eine Säule von $300 \mathrm{~cm}$ Länge und 3,5 cm Durchmesser benutzt. Als Sorptionsmittel diente Kieselgel für Säulenchromatographie (Fa. E. Merck, Darmstadt), Korngröße 0,05-0,2 mm, Menge: 400 g. Elutionsmittel: Benzol; Benzol/Chloroform 50:50 (v/v), Chloroform, Chloroform/Methanol-Gemisch (99,5:0,5 [v/v], 99:1 usw., jeweils eine Steigerungsrate von 0,5 Methanolanteil bei einer Minderungsrate von 0,5 Chloroformanteil bis zu dem Verhältnis 95:5). Danach betrug die Steigerungstate von Methanol - bis zu dem Verhältnis $90: 10$ - jeweils 1 bei einer entsprechenden Minderungsrate des Chloroformanteils von ebenfalls 1 . Anschließend wurden beide Lösungsmittel im Verhältnis 50:50 angewandt und zuletzt eluierten wir mit reinem Methanol.

Von jedem Elutionsmittel wurden 21 verwendet; die Fraktionen betrugen jeweils $200 \mathrm{ml}$. Diejenigen, die den Metabolit V enthielten (etwa $600 \mathrm{mg}$ ) wurden erneut an Kieselgel chromatographiert. Hierbei wurde eine Säule von $22 \mathrm{~cm}$ Länge und $2 \mathrm{~cm}$ Durchmesser benutzt. Das erhaltene Produkt enthielt Spuren des Metaboliten IV.

\section{Geräte}

Für die UV-Spektroskopie wurde ein Unicam-Leitz-Spektralphotometer SP 800 B benutzt. Die Spektren wurden in Chloroform und in Äthanol aufgenommen.

Die Aufnahme der IR-Spektren erfolgte mit einem Perkin-ElmerSpektralphotometer Modell 221 mit Gitter-Prisma-Austauscheinheit. Es wurde die $\mathrm{KBr}$-Preßtechnik angewandt. Die Aufnahme erfolgte unter Standardbedingungen $(1 \mathrm{mg}$ Substanz und $300 \mathrm{mg}$ $\mathrm{KBr}$ ).

Massenspektrometer:

a) niederauflösend $\mathrm{CH} 4$ (MAT)

b) hochauflösend MS 9 (AEI)

Ionisierungsenergie $70 \mathrm{eV}$

NMR-Spektren: Varian A 60.

\section{Ergebnisse}

Bei der Untersuchung der Urinproben zweier Patienten, die mehrere Wochen lang täglich $1,2 \mathrm{~g}$ Carbamazepin eingenommen hatten, ließen sich in Übereinstimmung mit früheren Mitteilungen $(1,2)$ außer dem unver- 


\section{Man muß die \\ Dinge \\ nurin den Griff \\ kriegen!}

Daß beispielsweise Thermometer so gut ablesbar sind wie Einschlußthermometer.

(Ohne so zerbrechlich zu sein). Und so robust wie Stabthermometer. (Ohne deren schwindsüchtige Graduierung).

Diese Eigenschaften haben Bistabil-EternaThermometer.

Mehr darüber auf der nächsten Seite

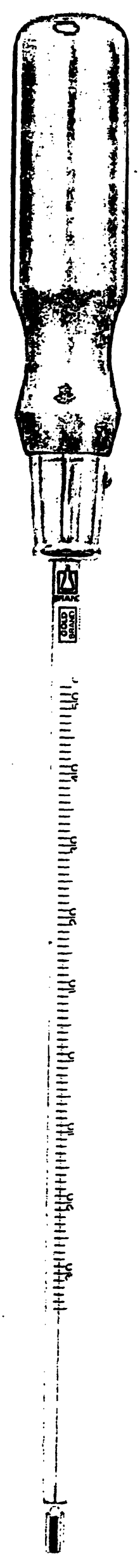

RUDOLF BRAND

LABORGERÄTE UND VAKUUMPUMPEN 698 WERTHEIM/MAIN · POSTFACH 310 

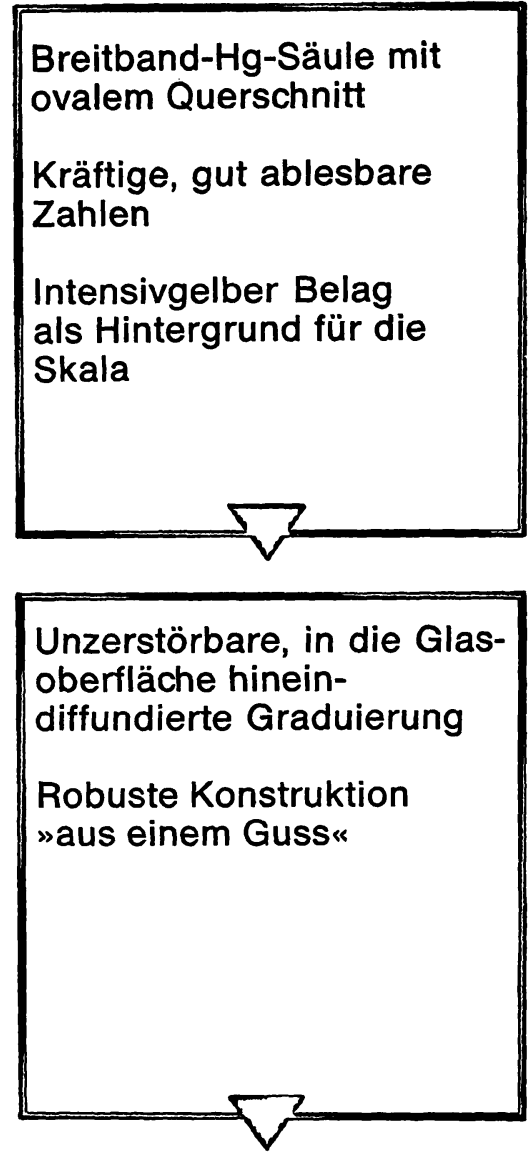

\section{Transparente Einzelver-} packung

Bestellnummer auf jedem Thermometer $\int_{\text {keit }}^{\text {Ausgezeichnete Ablesbar- }}$

Vielfache Lebensdauer bei voller Gebrauchstüchtigkeit

Kosten- und Zeitersparnis auch in der Lagerhaltung
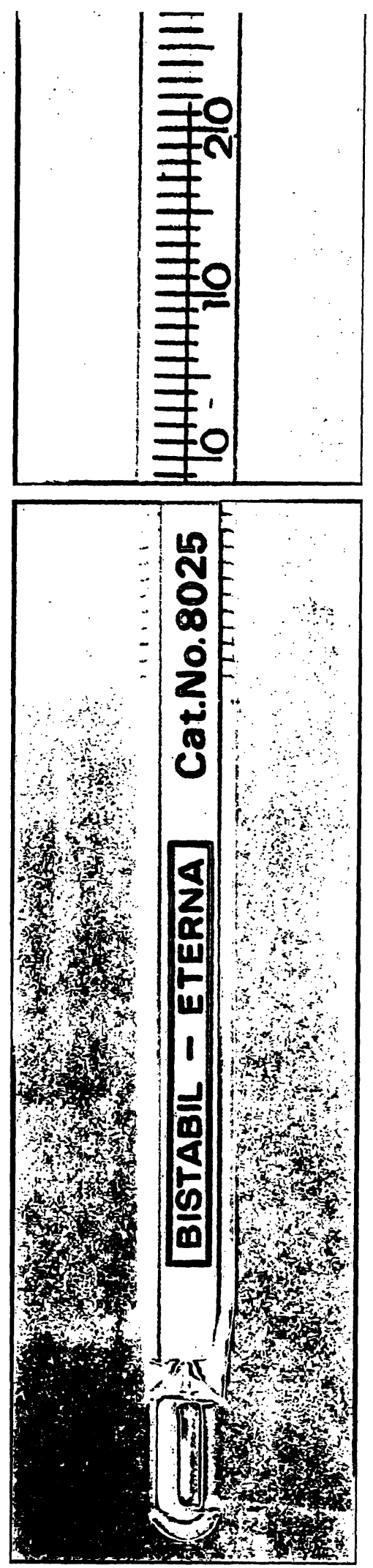

\section{COUPON}

BRAND-GERÄTE WEIL DIE RENTABILITÄT ENTSCHEIDET

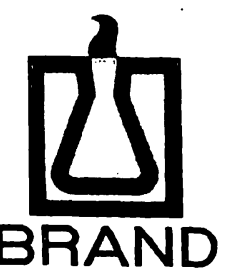

Hier konnte Ihnen natürlich nur eine Übersicht über einige wichtige Eigenschaften dieser Geräte gegeben werden. Wenn Sie aber diesen Abschnitt mit einer, notfalls unfrankierten, Postkarte an uns schicken, lassen wir Ihnen gerne weitere Informationen zukommen über:

BISTABIL-ETERNA-THERMOMETER

BRAND Fabrik für Laborgeräte 6980 Wertheim-2 
änderten Carbamazepin 7 Metaboliten dünnschichtchromatographisch nachweisen (Abb. 1).

171 Harn der beiden Patienten wurden mit Salzsäure (25proz.) auf $\mathrm{pH} 3$ gebracht und mit Chloroform extrahiert. Bei der wiederholten Chromatographie des Chloroformextraktes an Kieselgel eluierten wir mit

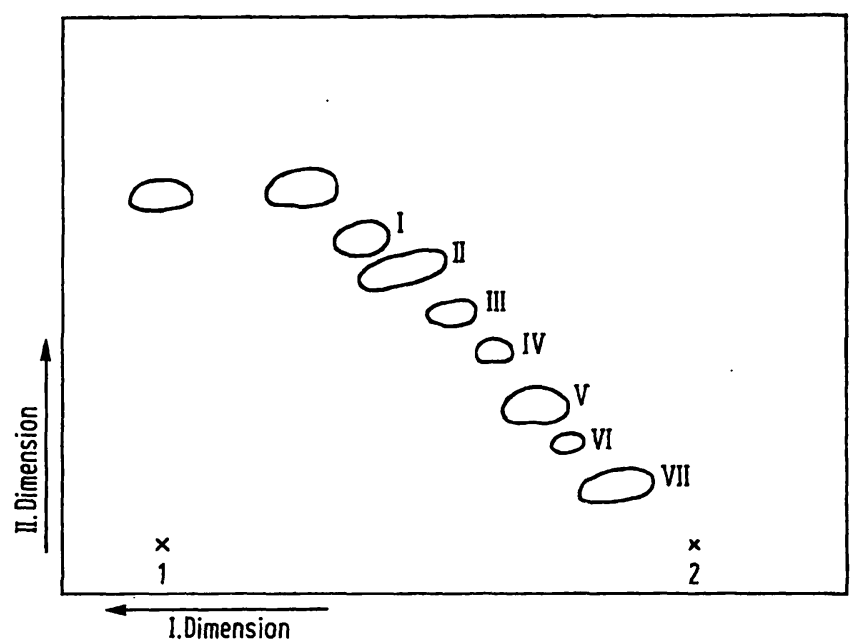

Abb. 1

Dünnschichtchromatogramm der Ausscheidungsprodukte des Carbamazepin nach zweidimensionaler Trennung. Sorptionsmittel Kieselgel G. Fließmittel: für beide Dimensionen Chloroform/Methano 90:10 (v/v). Nachweisreagenz: 70proz. Perchlorsäure; Fluoreszenzflecke unter der UV-Lampe. $1=$ Carbamazepin, $2=$ Harnextrakt

Chloroform/Methanol 95,5:4,5 (v/v) und 95:5 (v/v) den Metaboliten V. Nach Umkristallisation aus Chloroform/Petroleumbenzin (Kp $50-75^{\circ} \mathrm{C}$ ) wurde eine kristalline, dünnschichtchromatographisch einheitliche Substanz (Schmelzpunkt: $191-193^{\circ}$, unkorrigiert) erhalten.

Das UV-Spektrum wies - im Gegensatz zu Carbamazepin - keine Absorption auf. Dieses Verhalten zeigt, daß das Chromophor des Carbamazepin (Doppelbindung im Siebenerring in Konjugation mit beiden Benzolkernen) fehlt.
Wegen der sehr geringen Löslichkeit von Metabolit V in Chloroform mußte das IR-Spektrum in $\mathrm{KBr}$ aufgenommen werden. Im Infrarot waren im Bereich der gebundenen $\mathrm{OH}-\mathrm{Valenzschwingungen} \mathrm{starke} \mathrm{breite}$ Banden zu sehen, die zum Teil den Bereich der NHValenzschwingungen überdeckten. Das Vorliegen der Amidgruppe war jedoch durch die Amid I-Bande (nahe $1660 \mathrm{~cm}^{-1}$ ) zu erkennen. Im Bereich der Gerüstvalenzschwingungen traten die charakteristischen $\mathrm{Ab}$ sorptionen der aromatischen Doppelbindungen auf.

Durch hochauflösende Massenspektroskopie ließ sich ein Molekulargewicht von 270 ermitteln und die Summenformel $\mathrm{C}_{15} \mathrm{H}_{14} \mathrm{~N}_{2} \mathrm{O}_{3}$ eruieren.

Das NMR-Spektrum zeigte für die isolierte Substanz genau wie bei Carbamazepin den Signalkomplex für 8 aromatische Wasserstoffe bei $\gamma=2,5-2,8 \mathrm{ppm}$. Ein unscharfes Dublett befand sich bei $\gamma=4,15 \mathrm{ppm}$ für zwei Aryl-CH-OH-Gruppen. Bei $\gamma=5,25 \mathrm{ppm}$ und $\gamma=6,3 \mathrm{ppm}$ befand sich je ein Signal eines Wasserstoffes der $\mathrm{CONH}_{2}$-Gruppe. Folglich fehlten je zwei Olefinprotonen, wie sie bei Carbamazepin vorkommen und stattdessen liegen zwei Arylcarbinol-Gruppen vor.

Aus den spektroskopischen Befunden läßt sich für Metabolit V folgende Struktur ableiten (Abb. 2):<smiles>NC(=O)N1c2ccccc2C(O)C(O)c2ccccc21</smiles>

Abb. 2

Metabolit $\mathrm{V}=10,11$-Dihydroxy-5H-dibenzo-(b, f)-dihydroazepin-5carbamoyl

Herm Dr. H.-W. FehlHaber, Institut für Organische und Biochemie, möchten wir für die Ausführung und Deutung der Massen- und NMR-Spektren danken.

\section{Literatur}

1. Braunhofer, J. und L. Zicha, Med. Welt 17, 1875 (1966). 2. WeIst, F. und L. ZiChA, Arzneimittel-Forsch., Aulendorf 17, 874 (1967). - 3. Scheiffarth, F., F. Weist und L. Zicha, diese
Z. 4, 68 (1966). - 4. Christiansen, J., Scand. J. clin. Laborat. Invest. 27, Suppl. 118, 67 (1971).

Priv.-Doz. Dr. S. Goenechea

Institut für Gerichtliche Medizin der Universität Bonn 53 Bonn

Stiftsplatz 12 